\title{
La investigación educativa en el profesorado universitario: hacia una investigación basada en el diseño instruccional
}

\author{
Jorge Balladares-Burgos ${ }^{\mathrm{a}, *}$ \\ ${ }^{a}$ Universidad Tecnológica Equinoccial. Facultad de Comunicación, Artes y Humanidades. Carrera de Ciencias de la Educación.Rumipamba s/n entre \\ Bourgeois y Atahualpa. Quito, Ecuador.
}

PUNTOS DESTACADOS

- Problemática de la formación del profesorado en el desarrollo de competencias investigativas.

- La investigación educativa y su integración en la práctica docente.

- La Investigación basada en el Diseño como una opción para la investigación educativa.

- La Investigación basada en el Diseño integra la investigación con el diseño y la práctica.

- La investigación basada en el Diseño busca la transformación y mejoramiento de los procesos educativos.

INFORMACIÓN DEL ARTÍCULO

Historial del artículo:

Recibido el 20 de junio de 2018

Aceptado el 16 de septiembre de 2018

Publicado el 19 de septiembre de 2018

Palabras clave:

Investigación educativa

Investigación basada en el diseño

Profesorado universitario

Calidad educativa
RESUMEN

Hoy en día el profesorado universitario se enfrenta a nuevos desafíos en torno al mejoramiento de la calidad educativa e innovación en la educación superior. Uno de estos retos es la integración de la investigación con las actividades docentes y el diseño curricular. El presente artículo indaga sobre la problemática de integración de la investigación en la práctica docente en educación superior. A partir de una revisión de la literatura, se propone a la Investigación basada en el Diseño como un enfoque alternativo que proporciona los horizontes epistemológicos y metodológicos fundamentales para integrar la investigación con el diseño y la práctica. Este tipo de investigación permite incidir en contextos educativos inmediatos, logrando que los resultados de la investigación se concreticen y se apliquen; además, posibilita que el contexto y la práctica reformulen las teorías que sustentan la práctica educativa.

() 2018 Balladares-Burgos CC BY 3.0 EC

\section{Introducción}

Hoy en día el profesorado universitario tiene como desafío combinar su tarea docente con la investigación. El perfil del docente del siglo XXI conjuga estos dos componentes: no se puede pensar en un docente que no investigue, y no se puede plantear una investigación que no incida en el proceso de enseñanza-aprendizaje. En este sentido, el presente artículo plantea algunas pistas de la investigación educativa del profesorado universitario a partir de la Investigación basada en el Diseño.

Hoy en día es necesario repensar la educación superior en tiempos de una sociedad del conocimiento y de la información. Por años se ha presionado a las Universidades a expandir tanto su oferta académica y el libre acceso de nuevos estudiantes por las necesidades del mundo laboral. Sin embargo, la Universidad se enfrenta con industrias del conocimiento que proponen el desarrollo de competencias laborales inmediatas que un profesional necesita para responder a la sociedad del conocimiento; por ende, los profesionales graduados se cuestionan sobre el real beneficio de una educación universitaria versus lo que las nuevas industrias del conocimiento le ofrecen para su desarrollo

\footnotetext{
*Autor principal: Balladares-Burgos. Universidad Tecnológica Equinoccial. Facultad de Comunicación, Artes y Humanidades. Carrera de Ciencias de la Educación.Rumipamba s/n entre Bourgeois y Atahualpa. Quito, Ecuador. Correo electrónico: jballadares@ute.edu.ec
}

profesional. Es importante que las universidades incorporen la investigación y el desarrollo de competencias investigativas en su proceso educativo para responder las demandas laborales (Laurillard, 2002).

Se puede mencionar que entre las causas de esta problemática se encuentra la poca efectividad de los programas de capacitación para el desarrollo de competencias investigativas en el docente universitario. El profesorado universitario se encuentra en medio de la tensión y la polémica al percibir un cierto grado de insatisfacción respecto a la calidad de los procesos de enseñanza e investigación en su práctica docente. Hay que reconocer que la profesión docente se enfrenta a nuevos retos educacionales que responden al mundo laboral; entre esos retos, se promueve la incorporación de competencias investigativas que incidan en la cientificidad del conocimiento y mejoramiento de las prácticas docentes tanto dentro como fuera del aula ante las nuevas generaciones de estudiantes (Pérez, 2010; Cobo y Moravec, 2011; Balladares-Burgos, 2017).

El docente universitario se encuentra en la encrucijada de formarse en investigación para mejorar sus métodos y técnicas de enseñanza. En un contexto global, se reconoce que la investigación en las universidades ha fallado, en especial, con los estudiantes de grado. Varios estudiantes egresan de las universidades sin una experiencia sólida en investigación (Boyer, 1990). Los estudiantes no se encuentran involucrados en la generación del conocimiento, y 
se percibe que hay intentos aislados para la investigación (Huggins, Jenkins, y Scurry, 2007).

En el caso de la educación superior en el Ecuador, una de las dificultades del profesorado universitario en las últimas décadas es que sus horas de trabajo estaban dedicadas casi en su totalidad a la docencia, contando con poco tiempo para la investigación. Esta realidad también evidencia la falta de formación en investigación del docente de la educación superior. Los intentos de participar en proyectos de investigación y publicar en revistas científicas eran esporádicos, por lo que dentro de la percepción docente la investigación se volvía una utopía, un ideal difícil de concretar en su desarrollo profesional. Una mercantilización del conocimiento, la privatización de universidades versus el fortalecimiento de la educación superior como bien público, la falta de una profesionalización del docente universitario, entre otras, son algunas de las causas de que la investigación no fuera considerada como prioridad de las universidades por muchos años - al menos, en la práctica- (Quirola, 2010; Ramírez, 2016; Quindemil, Intriago y Rumbaut, 2017).

Para que el docente comprenda el cambio de la Educación Superior en el Ecuador, resulta imprescindible tomar conciencia del contexto universitario en la transición hacia una sociedad del Buen Vivir. Ciencia, Tecnología, Innovación y Conocimiento son ejes fundamentales para la transformación de la universidad ecuatoriana. Esta transición de la universidad en sociedad se basa en el derecho universal a la educación, el Plan Nacional para el Buen Vivir, la nueva Ley Orgánica de Educación Superior (LOES), e iniciativas locales y regionales para un nuevo tipo de sociedad justa, democrática e integrada a la naturaleza (Quirola, 2010).

\section{Metodología}

A partir de esta problemática global y local en la educación superior, surgen las siguientes preguntas: ¿es posible investigar a partir de la práctica docente universitaria? ¿Se puede generar una investigación científica en el aula? ¿Qué tipo de investigación integra el currículo y la práctica?

Dentro de la metodología del presente artículo, se realizó una revisión documentada de la literatura sobre el tema. El punto de partida para el presente artículo ha sido la revisión de la literatura de metodologías cualitativas, en especial, la Investigación basada en el Diseño (Design-Based Research). Se han revisado artículos a este enfoque metodológico de los últimos años a través de buscadores científicos.

De unos 20 artículos revisados, se han escogido 10 artículos que se enfocan en la variable Design-Based Research, Design Research, Investigación basada en el Diseño, Estudios del Diseño, y Diseño basado en la Investigación. A su vez, se ha complementado con literatura encontrada en bases de datos que se ha considerado como pertinente para el tema.

\section{Resultados}

Tal como se ha manifestado en el presente artículo, la investigación educativa es clave para el mejoramiento de procesos de enseñanza-aprendizaje en el aula. Aunque se adopten nuevas estrategias metodológicas para el desarrollo de la investigación en el aula, es importante incluir un horizonte epistemológico que oriente la investigación educativa. Para ello se propone como enfoque al paradigma de la Investigación basada en el Diseño (Design-Based Research o DBR, por sus siglas en inglés), que conjuga el diseño instruccional o pedagógico de un programa o curso con la investigación y la práctica. Este paradigma de investigación educativa contribuye a explicar de qué manera las innovaciones educativas funcionan en la práctica (Gibelli, 2014).

La Investigación basada en el Diseño permite que el investigador se inserte a un equipo de trabajo interdisciplinar y pueda influir en su contexto inmediato. Por ende, este enfoque busca la generación de innovaciones en contextos locales. Entre las ventajas que puede brindar este paradigma emergente para la investigación en tecnología educativa se pueden mencionar las siguientes:

- la posibilidad de explorar posibilidades de nuevos entornos del proceso de enseñanza-aprendizaje;

- desarrollar teorías contextualizadas sobre el proceso de enseñanza-aprendizaje;

- construir el conocimiento sobre diseño; y

- aumentar la capacidad humana para la innovación (The Design-Based Research Collective, 2003).

La Investigación basada en el Diseño conjuga el diseño con la investigación y la práctica. Este diseño está basado en el aprendizaje y se encuentra ligado a múltiples diseños y metodologías de la investigación, generando así una metodología híbrida (Wang \& Hannafin, 2005). Este tipo de investigación educativa se lo puede definir como una metodología sistemática y flexible que busca mejorar las prácticas educativas a través del análisis, diseño, desarrollo e implementación, basado en la colaboración entre investigadores y practicantes en una realidad concreta, y conduciendo hacia principios y teorías del diseño contextualizados (Wang \& Hannafin, 2005). Entre sus características se puede mencionar las siguientes:

- Los objetivos generales de los entornos del diseño del aprendizaje y desarrollo de teorías del aprendizaje.

- El desarrollo y la investigación se realizan en los ciclos continuos de aprendizaje, análisis y rediseño.

- La investigación sobre diseños debe generar teorías compartidas que ayuden a comunicar implicaciones relevantes a los docentes e investigadores.

- La investigación debe tomar en cuenta cómo funciona el diseño, no solo considerando sus debilidades sino analizando sus interacciones.

- El uso de métodos que pueden generar procesos para la acción de acuerdo a resultados de interés (The Design-Based Research Collective, 2003).

Wang y Hannafin (2005) mencionan los siguientes atributos del Diseño basado en Investigación:

a. Pragmatismo: en el que la teoría se alimenta de la práctica.

b. Fundamentación: el diseñó se basa en investigaciones, teoría y práctica relevantes.

c. Flexibilidad: el diseño es participativo, abierto y continuo. 
d. Integración: se integran diferentes métodos para maximizar la credibilidad de la investigación.

e. Contextualización: la investigación se inserta en un determinado entorno y sus necesidades.

Cabe indicar que una de las fortalezas de la Investigación basada en el Diseño es que ubica la investigación educativa en el contexto natural en el que ocurren los fenómenos estudiados, lo que produce cambios específicos en el contexto estudiado. Promueve la opción de enfoques sistémicos, usa variables que se tratan como interdependientes. Por último, este tipo de investigación tiene un carácter cíclico e iterativo de los diseños (Gibelli, 2014).

La Investigación basada en el Diseño es un estudio de campo que interviene en un contexto de aprendizaje particular, mediante un diseño instructivo, y logra una meta pedagógica definida. Las investigaciones generan conocimientos que contribuyan a mejorar la calidad del diseño y las prácticas instruccionales en diferentes niveles, contextos y áreas disciplinarias (Gibelli, 2014).

Entre los objetivos de la Investigación basada en el Diseño, se mencionan los siguientes:

a. No pretende controlar, sino identificar variables para caracterizar la situación.

b. No tiene como objetivo la replica de las implementaciones realizadas, sino la mejora del diseño implementado y la generación de pautas para la implementación de diseños educativos en situaciones similares.

c. No demuestra hipótesis, sino que desarrolla un perfil que caracterice el diseño en la práctica.

No obstante, los alcances de los resultados de una Investigación basada en el Diseño no solamente se circunscriben al ámbito de intervención, sino que permiten también generalizar o formular una nueva teoría.

Rinaudo y Dinolo (2010) mencionan tres fases para la preparación de una Investigación basada en el Diseño: preparación del diseño, implementación del diseño y análisis retrospectivo.

La primera fase consiste en la preparación del diseño de la investigación y tiene cuatro pasos. En el primer paso se definen las metas de aprendizaje de un programa o curso. Luego, en un segundo paso se realiza una descripción de las condiciones iniciales del contexto donde está inserto el programa o curso en estudio. Como parte de la preparación del diseño, el tercer paso consiste en la definición de las intenciones teóricas del estudio. Entre las teorías, se pueden distinguir las teorías macro, los marcos conceptuales y de acción, las teorías instructivas y las innovaciones ontológicas. Como cuarto paso de esta primera fase, se propone la elaboración de la intervención de la Investigación basada en el Diseño, con modos y medios para ejecutar el proceso, como son el reconocimiento de fuentes de datos y la elaboración de los instrumentos (DiSessa y Cobb, 2004; Rinaudo y Dinolo, 2010).

La segunda fase de la Investigación basada en el Diseño se ubica en la implementación de una metodología. En esta fase, se accede a las diferentes fuentes de información y se aplican diferentes técnicas de recopilación de la información (Gravemeijer y Cobb, 2006; Rinaudo y Donolo, 2010). A su vez, esta fase propone la realización de dos microciclos en secuencia iterativa.
El primer microciclo es el de diseño. En este primer microciclo se formulan una serie de conjeturas sobre la problemática del programa o curso. Para la formulación de las conjeturas, se revisan las condiciones iniciales del contexto instructivo.

El segundo microciclo es el de análisis. En este segundo microciclo se accede a las fuentes de datos y se recopila información a través del uso de técnicas e instrumentos. En esta fase el investigador inicia los análisis del levantamiento de la información in situ, es decir, en el lugar donde se realiza el estudio del programa o curso (Balladares-Burgos, 2018).

Tras la preparación y la implementación del Diseño basado en la Investigación que corresponde a la primera y segunda fases, se realiza una tercera fase que consiste en el análisis retrospectivo. De acuerdo a Rinaudo y Donolo (2010), esta fase comprende un análisis de los datos recogidos en las fases anteriores y una reconstrucción de la teoría instructiva elaborada durante la primera fase sobre la preparación del diseño.

Este análisis retrospectivo de datos recolectados se realizará de manera iterativa. Se sugiere llevar a cabo un primer ciclo de análisis desde lo cronológico. Luego, un segundo ciclo de análisis a partir de interpretaciones, conjeturas y refutaciones de los datos recopilados. Además del análisis que se pueda realizar a partir de las indicaciones sugestivas, un segundo ciclo de análisis nos permitirá establecer categorías para la interpretación de datos (Balladares-Burgos, 2018).

Las indicaciones sugestivas requieren de definición de variables o categorías; ellas son indispensables para poder establecer la tercera fase de la Investigación basada en el Diseño. Collins, Joseph y Bieleaczyc (2004) proponen las siguientes variables independientes a ser consideradas en un análisis retrospectivo: características de la institución, estudiante, recursos, desarrollo docente, financiamiento e implementación. Como variables dependientes se consideran las variables de clima escolar, de aprendizaje, y de sentido de comunidad o pertenencia.

Después del análisis de datos recolectados y de la definición de categorías o variables, se realiza una reconstrucción de la teoría instructiva a partir de la revisión de intenciones teóricas en relación a los resultados de análisis. Según Rinaudo y Donolo (2010) el propósito de esta etapa es la reconstrucción de la teoría instructiva luego de haber analizado e interpretado los datos surgidos en la revisión del diseño. En esta fase se pueden realizar reajustes en los argumentos y fundamentos teóricos que se plantearon en la primera fase. A su vez, se puede realizar un examen de las intenciones teóricas más amplias del estudio, y establecer las contribuciones logradas por la investigación.

Para mantener la calidad de la investigación basada en el Diseño, Rinaudo y Donolo (2010) proponen cuatro indicadores de calidad:

a. calidad de la teoría,

b. relevancia de las metas pedagógicas,

c. rigurosidad del diseño,

d. coherencia en la reconstrucción del estudio.

Estos indicadores buscan asegurar la calidad del la Investigación basada en el Diseño con el fin de lograr los resultados cualitativos esperados. 
Tabla 1

Detalle de fases y tareas de la Investigación basada en el Diseño

\begin{tabular}{|c|c|}
\hline Fases & Tareas del DBI - Descripción \\
\hline $\begin{array}{l}\text { Fase } 1 \\
\text { Preparación } \\
\text { diseño }\end{array}$ & $\begin{array}{ll}- & \text { Definición metas de aprendizaje. } \\
- & \text { Descripción de las condiciones iniciales del } \\
& \text { contexto. } \\
- & \text { Definición de las intenciones teóricas. } \\
- & \text { Elaboración de intervención. } \\
- & \text { Definición de fuentes de datos. Elaboración de } \\
& \text { instrumentos. }\end{array}$ \\
\hline $\begin{array}{l}\text { Fase } 2 \\
\text { Implementación }\end{array}$ & $\begin{array}{ll}\text { - } & \text { Microciclo de diseño: problemática. } \\
\text { - } & \text { Microciclo de análisis. }\end{array}$ \\
\hline $\begin{array}{l}\text { Fase } 3 \\
\text { Análisis retrospec- } \\
\text { tivo }\end{array}$ & $\begin{array}{ll}\text { - } & \text { Análisis de datos recolectados. } \\
\text { - } & \text { Indicaciones sugestivas. } \\
\text { - } & \text { Reconstrucción de las intenciones teóricas. } \\
\text { - } & \text { Conclusiones y recomendaciones. }\end{array}$ \\
\hline
\end{tabular}

La clave de una Investigación basada en el Diseño es que sus resultados tengan una incidencia en el mejoramiento o rediseño del programa o curso. Esto garantiza que el diseño y la investigación se retroalimente de la práctica, y a su vez, que la práctica se transforme en la búsqueda de la calidad educativa. Permite también conocer los factores de éxito o fracaso de las innovaciones educativas.

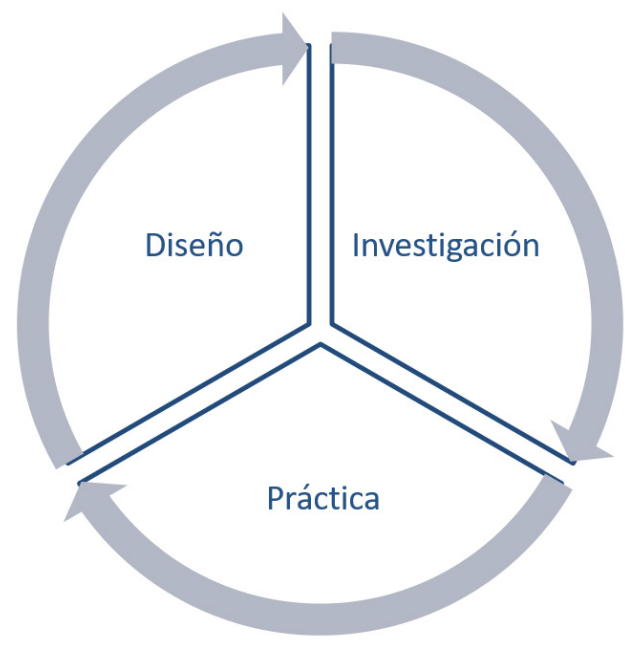

Fig. 1. Componentes de la Investigación basada en el Diseño.

\section{Conclusiones}

Ante la problemática de la incorporación de la investigación educativa en la práctica docente universitaria, se presenta el enfoque de la Investigación basada en el Diseño como una alternativa de investigación sobre los diseños pedagógicos, diseños curriculares, diseños de cursos, diseños de programas y carreras, además de la utilización de recursos y material pedagógico. Este enfoque permite integrar la investigación con el diseño y la práctica y, a su vez, logra incidir en la transformación de la práctica en sí.

El impacto que genera este tipo de investigación permite incidir en el contexto de un curso, programa o proyecto educativo en miras a mejorar la propuesta. Además explora futuras posibilidades de implementar programas o proyectos similares. También propone teorías contextualizadas sobre el proceso educativo digital. Finalmente, indaga potenciales formas de innovación en la educación superior.
A través de este tipo de enfoques, el docente puede activarse en la investigación e integrarla a su práctica docente, al diseño curricular e instruccional y en los contextos en los que se encuentre inmerso. La integración de la investigación en el quehacer del profesorado universitario pretende mejorar la calidad del proceso enseñanzaaprendizaje en el aula en búsqueda de la excelencia, la innovación y la pertinencia. La investigación también deberá ser transferida a la sociedad en función de responder a las necesidades del entorno socio-cultural.

Es importante considerar que los programas de formación del profesorado universitario incorporen el desarrollo de competencias investigativas. Para ello, investigación, docencia y diseño son componentes distintos pero complementarios en su interacción que pueden ser fuente de información clave para comprender procesos innovadores en la educación. De esta manera, la investigación del docente de educación superior no solamente se enfocará hacia la investigación científica en su disciplina o área de conocimiento, sino que también se enfocará en su quehacer pedagógico como investigación educativa.

En los últimos años hubo una tendencia sobre el uso exclusivo de enfoques cuantitativos en la investigación educativa. El ámbito educativo, al ser un ámbito con sujetos educativos como actores que poseen niveles de relaciones humanas, desafían a la investigación a desarrollar metodologías cualitativas que permitan explicar y comprender elementos complejos del hecho educacional en los seres humanos. En este sentido, se propone a la Investigación basada en el Diseño como una alternativa metodológica no solamente de enfoque cualitativo sino también de enfoque mixto.

Este tipo de investigación permite incidir en contextos inmediatos, es decir, que los resultados de la investigación se concreticen y se apliquen en los diferentes contextos educativos, y a su vez, que el contexto y la práctica permitan reformular las teorías que sustentan la práctica educativa. Permite enfocarse tanto en la enseñanza como en el aprendizaje, y a su vez, tiene la sistematización y la flexibilidad necesaria para ligarse a diferentes diseños y a una diversidad de metodologías, técnicas e instrumentos de la investigación. Esta capacidad híbrida de la Investigación basada en el Diseño hace que este tipo de metodología se adapte a las necesidades de la investigación, al contexto investigado y a la realidad del investigador.

\section{Bibliografía}

Balbo, J. (2008). Formación en competencias investigativas, un nuevo reto en las universidades. Caracas: Universidad Central de Venezuela.

Balladares-Burgos, Jorge (2017). Una ética digital para las nuevas generaciones digitales. Revista PUCE, 104.

Balladares-Burgos, Jorge (2018). Diseño pedagógico de la educación digital para la formación del profesorado. RELATEC. Revista Latinoamericana de Tecnología Educativa, 17(1), 41-60. http:/ / doi.org/10.17398/1695-288X.17.1.41

Boyer, E. (1990). Scholarship reconsidered: priorities of the Professoriate. New Jersey: Carnegie Foundation for the Advancement of Teaching.

Cobo, C., y Moravec, J. (2011). Aprendizaje Invisible. Hacia una nueva ecología de la educación. Barcelona: Publica- 
cions i Edicions de la Universitat de Barcelona.

Collins, A., Joseph, D. y Bielaczyc, K. (2004). Design research: theoretical and methodological issues. Journal of the Learning Sciences, 13(1), 15-42. https://doi.org/10.1207/ s15327809j1s1301_2

DiSessa, A. y Cobb, P. (2004). Ontological innovation and the role of theory in design experiments. Journal of the Learning Sciences, 13(1), 77-103. https:/ / doi.org/10.1207/ s15327809jls1301_4

Gibelli, T. (2014). La investigación basada en diseño para el estudio de innovación en educación superior que promueve la autorregulación del aprendizaje utilizando TIC. Congreso Iberoamericano de Ciencias, Tecnología, Innovación y Educación, 2-16.

Gravemeijer, K., y Cobb, P. (2006). Design research from the learning design perspective. In Van den Akker, J., Gravemerijer, K., McKenney, S., \& Nieveen, N. (Eds.), Educational Design Research. London: Routledge.

Huggins, R., Jenkins, A., y Scurry, D. (2007). Undergraduate research in selected US universities: report on US visit-institutional case studies. York, UK: Higher Education Academy.

Laurillard, D. (2002). Rethinking Teaching for the knowledge society. Educase review, 1(37), 16-25.

Lodico, M., Spaulding, D., y Voegtle, K. (2010). Methods in educational research: from theory to practice. San Francisco, CA: Jossey-Bass.

Pérez, A. (2010). Nuevas exigencias y escenarios para la profesión docente en la era de la información y de la incertidumbre. Revista Interuniversitaria de Formación del Profesorado, 68, 17-36.

Pérez Serrano, G. (2003). Pedagogía social, educación social: Construcción científica e intervención práctica. Madrid: Narcea.

Quindemil, E., Intriago S. y Rumbaut, F. (2017). La educación superior en el Ecuador en el período 2008-2016. oportunidades para la universidad técnica de Manabí. Revista Cognosis, 2(3), 71-84.

Quirola, D. (2010). La universidad ecuatoriana en la transición hacia la sociedad del buen vivir basada en el bioconocimiento. En R. Ramírez, Transformar la Universidad para transformar la sociedad (pp. 195-216). Quito: Senplades.

Ramírez, R. (2016). Universidad urgente para una democracia emancipada. CIESPAL: Quito.

Rinaudo, M., y Donolo, D. (2010). Estudios de diseño. Una perspectiva prometedora en la investigación educativa. Revista de Educación a Distancia, 22, 1-29.

The Design-Based Research Collective. (2003). Design-Based Research: An Emerging Paradigm for Educational Inquiry. Educational Researcher, 32(1), 5-8. https:/ / doi.org/10.3102/0013189X032001005

Valverde, J. (2014). La definición del tema de investigación. Plan de Formación de Doctores en Tecnología Educativa. Cáceres: Universidad de Extremadura.

Wang, F., \& Hannafin, M. (2005). Design-based research and Technology-Enhanced Learning Environments. ETRED, 53(4), 5-23. https:/ / doi.org/10.1007/BF02504682

Copyright: (C) 2018 Balladares-Burgos. Este es un artículo de acceso abierto distribuido bajo la licencia Creative Commons de Atribución 3.0 de Ecuador, que permite su uso sin restricciones, su distribución y reproducción por cualquier medio, siempre que el trabajo original sea fielmente citado. 